\title{
Endoscopic discrimination of sessile serrated adenomas from other serrated lesions
}

\author{
SHIN HASEGAWA ${ }^{1}$, KEIICHI MITSUYAMA ${ }^{1}$, HIROSHI KAWANO ${ }^{1}$, KEIKO ARITA ${ }^{1}$, YASUHIKO MAEYAMA ${ }^{1}$, \\ YOSHITO AKAGI ${ }^{2}$, YASUTOMO WATANABE ${ }^{1}$, YOSHINOBU OKABE ${ }^{1}$, OSAMU TSURUTA $^{1}$ and MICHIO SATA ${ }^{1}$ \\ ${ }^{1}$ Division of Gastroenterology, Department of Medicine and ${ }^{2}$ Department of Surgery, \\ Kurume University School of Medicine, Kurume 830-0011, Japan
}

Received December 17, 2010; Accepted June 16, 2011

DOI: $10.3892 / \mathrm{ol} .2011 .341$

\begin{abstract}
Sessile serrated adenoma (SSA) is a proposed precursor of colorectal carcinogenesis. This study aimed to analyze the potential of endoscopy to discriminate SSA from other serrated lesions, specifically traditional serrated adenoma (TSA) and hyperplastic polyp (HP). Of 145 serrated lesions, 111 sessile serrated lesions including 32 TSAs, 25 SSAs and 54 HPs were analyzed for size, color, location and morphologic features using conventional endoscopy and magnifying chromoendoscopy. SSA was preferentially located in the right colon, whereas TSA and HP were located in the left colon. The sizes of SSA and TSA were larger than those of HP. The lesion color was indistinguishable among TSA, SSA and HP. Macroscopically, a pinecone-like or twotier raised appearance were found more frequently in TSA than in SAA and HP. Under magnified chromoendoscopic observation, the stellar $\mathrm{III}_{\mathrm{L}}$ pit pattern and fern-like appearance were observed more frequently in TSA than in SAA and HP. In conclusion, endoscopic discrimination between SSA and other sessile serrated lesions based on morphological features was difficult. However, size and location of the lesions facilitated diagnosis.
\end{abstract}

\section{Introduction}

Serrated polyps of the colorectum have been the focus of critical reappraisal and intense study in recent years. Previously, the majority of serrated, non-adenomatous polyps were diagnosed as hyperplastic polyps (HPs) and treated

Correspondence to: Dr Keiichi Mitsuyama, Division of Gastroenterology, Department of Medicine, Kurume University School of Medicine, Asahi-machi 67, Kurume 830-0011, Japan

E-mail: ibd@med.kurume-u.ac.jp

Abbreviations: SSA, sessile serrated adenoma; TSA, traditional serrated adenoma; HP, hyperplastic polyp; MSI, microsatellite instability; H\&E, hematoxylin and eosin

Key words: colon, endoscopy, serrated polyp, sessile serrated adenoma, traditional serrated adenoma, hyperplastic polyp as innocuous, benign lesions (1). Subsequently, HP was implicated in the development of colorectal cancer through a putative HP-serrated adenoma-colorectal cancer sequence. At present, the serrated polyps are regarded as heterogeneous lesions with several histological subtypes, including HP, traditional serrated adenoma (TSA) and sessile serrated adenoma (SSA) $(2,3)$.

Mounting evidence shows that SSA may be the precursor lesion through the previously described 'serrated pathway' of colorectal carcinogenesis (4), and some investigators recommend that SSA should be clinically managed in a similar manner to conventional adenomas (5). A greater frequency of microsatellite instability has been shown in SSA versus HP or TSA (6). SSA shows a higher frequency of CpG island methylation than conventional HP (7), while showing a higher frequency of $B R A F$ gene mutations and a lower frequency of $K$-ras gene mutations versus conventional adenoma $(8,9)$. Thus, reliable identification of SSA and its differentiation from other serrated lesions, such as HP and TSA, has important clinical implications and may condition therapeutic attitudes and endoscopic follow-up.

Colonoscopy is the only technique currently available that offers the potential to detect and remove colorectal lesions throughout the large intestine. Some authors previously reported the endoscopic features of TSA and HP (10-13). However, the endoscopic features of SSA have yet to be established. This study aimed to compare the endoscopic features of the three sessile serrated lesions, TSA, SSA and HP, and to determine whether SSA can be distinguished from other serrated lesions.

\section{Materials and methods}

Patient selection and study design. The present study was a retrospective analysis of a cohort of 101 patients $(73$ males and 28 females with a mean age of 62.5 years) who underwent total colonoscopy at the Kurume University Hospital, Japan, between January 2005 and September 2008 and were found to have 141 histologically proven serrated lesions. Of the 141 serrated lesions, 34 were found to be of the protruded form and 107 were of the sessile form (Table I). We focused on the 107 sessile lesions, which included 31 TSAs, 22 SSAs and 54 HPs. 
Table I. Relationship between overall shape and type of serrated colorectal lesion.

\begin{tabular}{lrrrr}
\hline & $\mathrm{n}$ & $\mathrm{HP}(\%)$ & SSA $(\%)$ & TSA (\%) \\
\hline Protruded type & 34 & $6(18)$ & $0(0)$ & $28(82)$ \\
Sessile type & 107 & $54(50)$ & $22(21)$ & $31(29)$ \\
\hline
\end{tabular}

HP, hyperplastic polyp; SSA, sessile serrated adenoma; TSA, traditional serrated adenoma. In the protruded lesion, the cancer component was included in 1 case of TSA, but none were included in HP. In the sessile lesion, the cancer component was included in 1 case of TSA and in 3 cases of SSA, but none were included in HP.

Endoscopic procedure. Prior to colonoscopy, 2 litres of a preparatory solution of electrolytes and polyethylene glycol was administered orally to each patient. The colonoscopies were performed using commercially available videocolonoscopes (CF-H260Z, CF-Q240Z; Olympus Optical Co., Tokyo, Japan) that provided conventional and magnified images. Lesions detected at colonoscopy were diagnosed by conventional endoscopy and magnifying chromoendoscopy with $0.075 \%$ crystal violet staining to observe pit patterns on the surface of the lesions. Chromoendoscopy was performed after the lesion was identified by conventional endoscopy. The endoscopic features were recorded during endoscopy. Endoscopically or surgically resected tissue specimens were mounted on a plate and fixed in $10 \%$ formalin for $24 \mathrm{~h}$. The specimens were washed with water and stained with Carrazzi's hematoxylin, and then images were captured.

Conventional endoscopic analysis. Images were evaluated by three endoscopists. Conventional endoscopic findings were classified based on size, color, location and macroscopic appearance, including pinecone-like appearance (13) (Fig. 1A) and two-tier raised appearance (Fig. 1B), both of which are characteristic of TSA. The two terms were determined after reviewing the images.

Pit pattern analysis. Using magnifying chromoendoscopy, pit patterns were classified based on the classification of Kudo et al (14) and Tsuruta et al (15). Type I are regular round crypts, type II are stellar or papillary crypts, type III are small tubular or roundish crypts $\left(\mathrm{III}_{\mathrm{S}}\right)$ or large tubular or roundish crypts $\left(\mathrm{III}_{\mathrm{L}}\right)$, type IV consists of branch- or gyrus-like crypts, and type $\mathrm{V}$ consists of irregular crypts $\left(\mathrm{V}_{\mathrm{I}}\right)$ or non-structural crypts $\left(\mathrm{V}_{\mathrm{N}}\right) . \mathrm{V}_{\mathrm{I}}$ was further divided into the well-demarcated subtype (showing clear pit contours) or the poorly demarcated subtype (without clear pit contours). Although not included in the above pit pattern classification, we also examined the presence of the stellar III $_{\mathrm{L}}$ pit pattern (Fig. 2A) and fern-like appearance (13) (Fig. 2B), which are characteristic pit patterns of TSA.

Histopathological analysis. Tissues were fixed in formalin and embedded in paraffin. Sections $(4 \mu \mathrm{m})$ were stained with hematoxylin and eosin (H\&E). Two gastrointestinal pathologists blinded to the clinical and demographic data classified each lesion as HP, SSA or TSA based on the criteria established by Torlakovic et al $(2,16,17)$ and other authors $(1,5,18)$. Lesions diagnosed as 'mixed' HP-tubular adenoma or SSA-tubular adenoma were excluded. The conventional criteria for HP included narrow straight crypts with a normal distribution of the proliferative zone in the base of the crypts with uniform maturation and serration toward the lumen of the colorectum. SSA was defined by architectural distortion with irregular dilated crypts, including dilatation of the base of the crypts that often have a boot-, L- or inverted T-shape; serration at the base of the crypts; and abnormal proliferation and maturation with mature goblet or foveolar cells at the base of the crypts. TSA was defined by an overall protuberant growth pattern with elongated viliform projections lined with atypical cells that exhibited elongated nuclei and eosinophilic cytoplasm. Representative histological features of each type of sessile serrated lesion are shown in Fig. 3.

Statistical analysis. The results were analyzed using SPSS statistical software. Size was expressed as the means \pm standard error (SE). The Student's t-test, Mann-Whitney U-test, Wilcoxon's signed-rank test, Pearson's correlation test, Spearman's correlation test and $\chi^{2}$ test were used as required. For all studies, $\mathrm{p}<0.05$ was used to determine statistical significance.

\section{Results}

Table I shows the relationship between overall shape and the histological type of the serrated colorectal lesions. Of the 141 lesions in total, 34 were found to be protruded lesions and 107 sessile lesions. In the protruded lesions, TSA was found in $82 \%$ and HP in $18 \%$ of lesions. In the sessile lesions, HP was found in $50 \%$, TSA in $29 \%$ and SSA in $22 \%$ of lesions. Of the protruded and sessile serrated lesions, the cancer component (intramucosal cancer) was present in $3.4 \%(2 / 59)$ of TSA, $13.6 \%(3 / 22)$ of SSA and $0 \%(0 / 60)$ of HP.

The association between clinicopathological variables and type of sessile lesion is shown in Fig. 4. Lesion size in TSA and SSA was larger than that of HP, with the mean values being 13.0, 14.2 and $6.2 \mathrm{~mm}$, respectively $(\mathrm{p}<0.01)$. Lesion size in TSA was comparable with that in SSA. Although the reddish lesion tended to increase in size in TSA, no significant difference in color was found between TSA, SSA and HP. Notably, SSA was preferentially located in the right colon compared with the left colon (81.8 versus $18.2 \%$ ), whereas TSA and HP were located in the left colon as compared with the right colon (61.3 versus $41.9 \%$, and 55.6 versus $44.4 \%$, respectively).

With regard to the macroscopic features (Fig. 5), the pinecone-like appearance was observed preferentially in TSA $(12.9 \%)$ compared with SSA $(0 \%, \mathrm{p}=0.0259)$ and HP (1.9\%, $\mathrm{p}=0.0259)$. Similarly, the two-tier raised appearance 

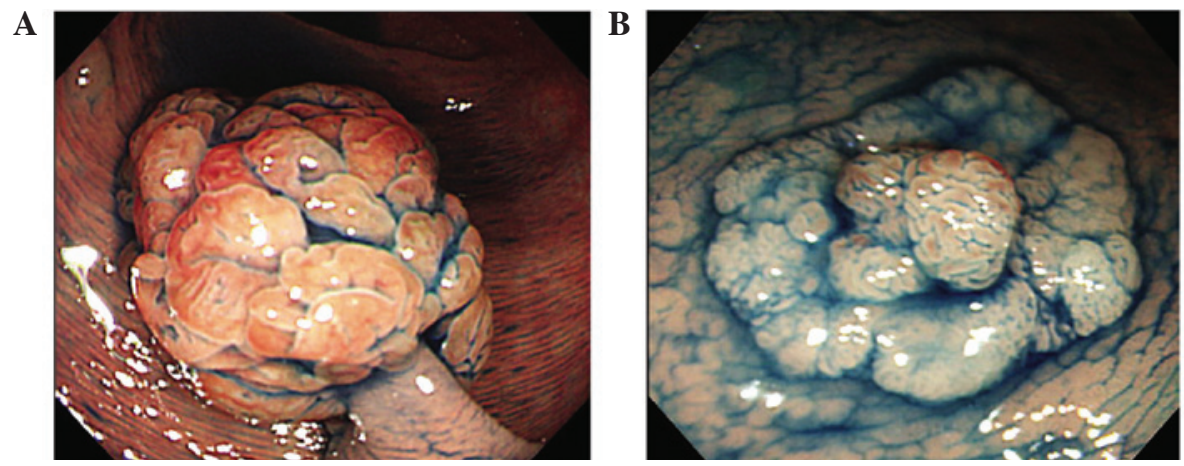

Figure 1. Endoscopic view of sessile serrated lesions after indigo carmine dye spraying. (A) Pinecone-like appearance. (B) Two-tier raised appearance. A granular protruded area is present on the flat part of the elevated lesion.

A

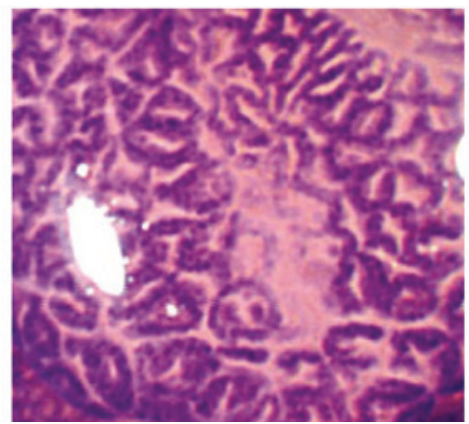

B

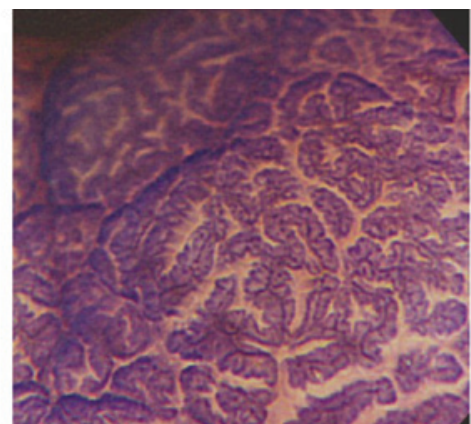

Figure 2. Magnified microscopic view of sessile serrated lesions with crystal violet staining. (A) Stellar $\mathrm{III}_{\mathrm{L}}$ pit pattern. (B) Fern-like appearance.
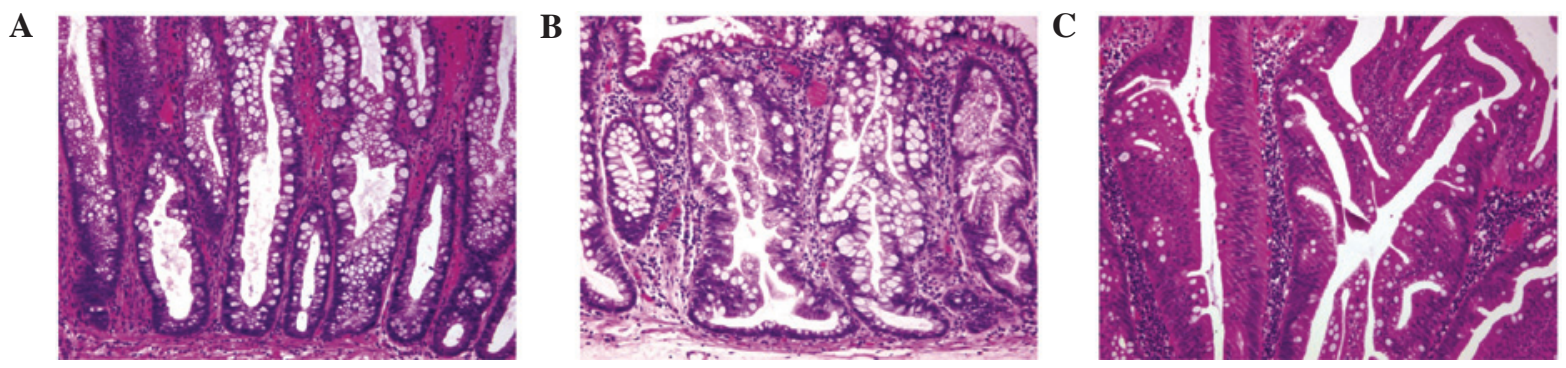

Figure 3. Representative histological features of (A) HP, (B) SSA and (C) TSA. H\&E; magnification, x200.

was found more frequently in TSA $(38.7 \%)$ than SSA $(9.1 \%$, $\mathrm{p}=0.0257)$ and $\mathrm{HP}(1.9 \%, \mathrm{p}<0.0001)$.

The ability to distinguish the type of sessile lesions by magnifying chromoendoscopy was investigated based on the pit pattern classification (Fig. 6). Information was available for a total of 33 lesions. In HP, $75.0 \%$ of the lesions were $\mathrm{III}_{\mathrm{L}}$ pit and $25.0 \%$ II pit; in SSA, $72.7 \%$ were III $_{\mathrm{L}}$ pit and $27.3 \%$ II pit; and in TSA, $100 \%$ were $\mathrm{III}_{\mathrm{L}}$ pit. However, the difference in pit patterns among TSA, SSA and HP was not statistically significant. In addition, $\mathrm{III}_{\mathrm{L}}$ pit in the three types of lesions was associated with the stellar pit in addition to the typical $\mathrm{III}_{\mathrm{L}}$ pit observed in tubular adenomas (termed stellar $\mathrm{III}_{\mathrm{L}}$ pit). Although not documented in the original pit pattern classification, the fern-like appearance, a unique pit for TSA, was observed more frequently in TSA (90\%) than in SSA (36.4\%, $\mathrm{p}=0.0237)$ and $\mathrm{HP}(8.3 \%, \mathrm{p}=0.0003)$. In addition, intramucosal cancer, which was present in a small proportion of TSA and SSA lesions, was not detectable macroscopically during endoscopy.

\section{Discussion}

The hypothesis of a serrated carcinogenetic pathway has been increasingly accepted $(5,19)$. Based on this hypothesis, a group of sporadic colorectal cancers are capable of originating in serrated lesions, mainly SSA. Approximately 8-15\% of all colorectal cancers may have originated from SSA (20). Therefore, it is important to accurately identify SSA and differentiate it from those lesions with less malignant potential, such as HP and TSA. As such, we investigated the endoscopic appearance of sessile serrated lesions and determined for the first time whether SSA can be distinguished from other serrated lesions.

First, the association between incidence of cancer and type of serrated lesion was investigated. Notably, of all the protruded or sessile serrated lesions, the cancer component was detected more frequently in SSA (13.6\%) than TSA (3.4\%) and HP (0\%), supporting the hypothesis that SSA, rather than TSA and HP, possesses a significant risk of subsequent colorectal cancer 
A

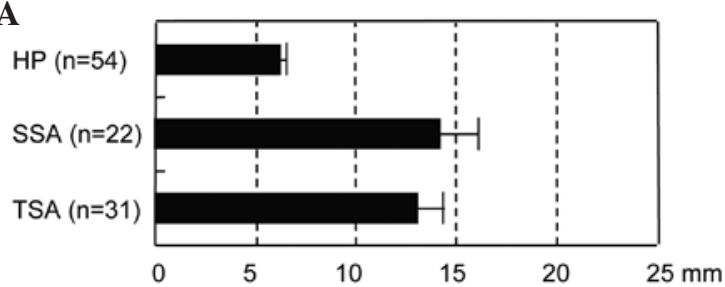

B

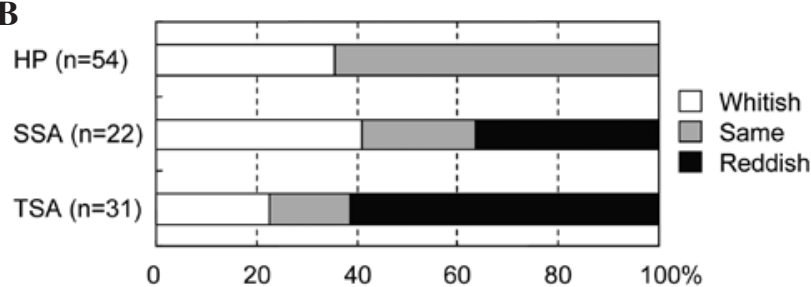

C

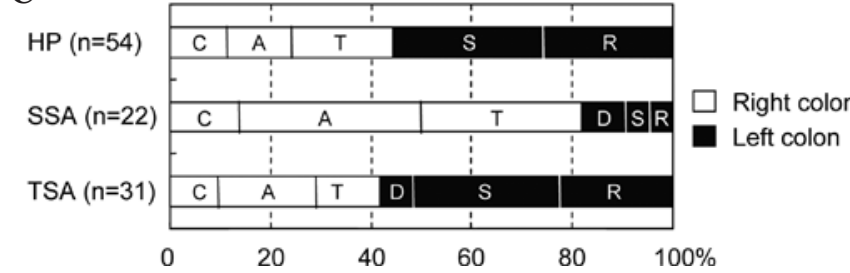

Figure 4. (A) Size, (B) color and (C) location in relation to the type of essile colorectal lesion. C, cecum; A, ascending colon; $\mathrm{T}$, transverse colon; $\mathrm{D}$, descending colon; $\mathrm{S}$, sigmoid colon; $\mathrm{R}$, rectum. The size was expressed as mean $\pm \mathrm{SE}$.

\section{A}

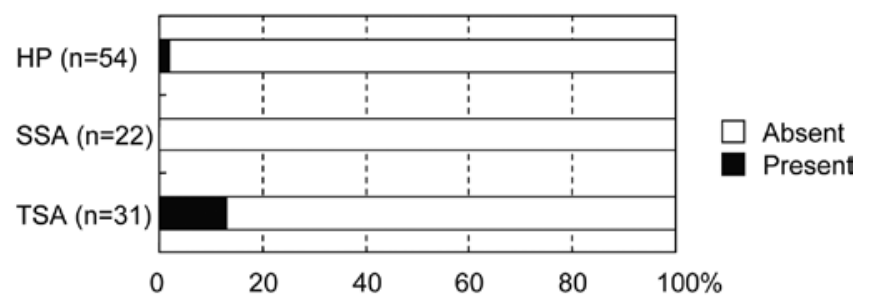

B

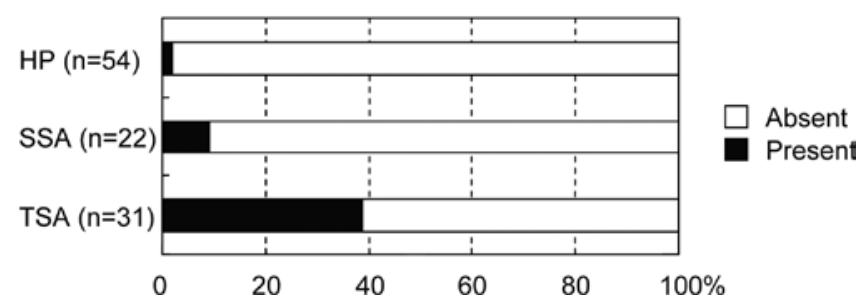

Figure 5. Macroscopic appearance in relation to the type of sessile colorectal lesions. (A) Pinecone-like appearance. (B) Two-tier raised appearance.

development. However, the retrospective nature of this study renders it difficult to draw satisfactory results as reliable as those that may have been achieved from a prospective study. We therefore believe that the main results of this study are plausible and at least show that additional prospective studies are required.

With regard to the clinicopathological features, the size of the lesions was larger in SSA and TSA than in HP (mean size $14.2,13.0$ and $6.2 \mathrm{~mm}$, respectively). Notably, SSA was located predominantly in the right colon, whereas HP and
A

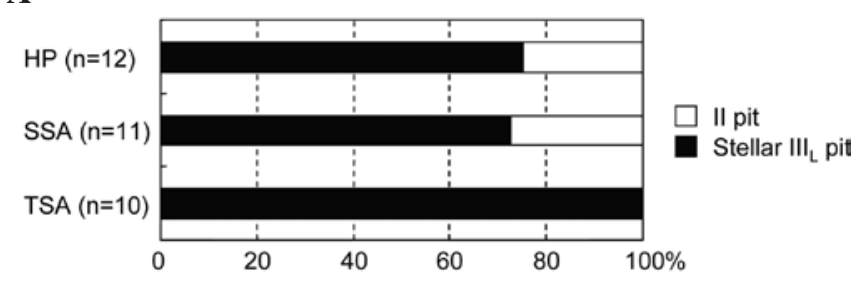

B

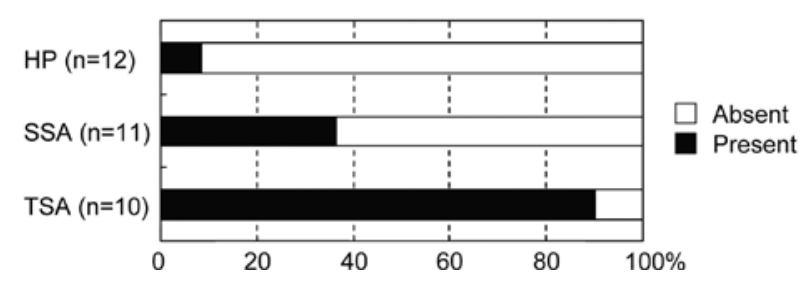

Figure 6. Pit pattern classification in relation to the type of sessile colorectal lesions. (A) Stellar $\mathrm{III}_{\mathrm{L}}$ pit pattern. (B) Fern-like appearance.

TSA were located in the left colon. These data were in agreement with previous studies, showing that SSA is larger than $10 \mathrm{~mm}$ in size and is confined to the right colon $(2,18)$. No color difference was found between TSA, SSA and HP. A lesion size larger than $10 \mathrm{~mm}$ and location in the right colon may represent criteria that facilitate distinguishing SSA from other serrated lesions.

For the macroscopic features, Sano et al have documented the pinecone-like appearance as a characteristic feature of TSA (13). In the present study, this feature was observed at a frequency of $12.9 \%$ in TSA, but was rarely observed in SSA and HP ( 0 and $1.9 \%$, respectively), which clearly supports the findings of Sano et al. Notably, the two-tier raised appearance, which is a new finding that has not previously been reported, was observed at a frequency of $38.7 \%$ in TSA but less frequently in SSA (9.1\%) and HP (1.9\%), indicating another possible feature of TSA. Although not so frequently observed, TSA has certain unique features that differentiate it from other serrated lesions. However, no such features were found for SSA using this approach.

Magnifying chromoendoscopy offers detailed analysis of the morphologic architecture of mucosal crypt orifices based on the pit pattern classification. However, HP, SSA and TSA were indistinguishable by this pattern of classification since all three lesions had II or stellar $\mathrm{III}_{\mathrm{L}}$ pit patterns with similar populations. A previous report proposed that a fern-like appearance was characteristic of TSA with a high diagnostic accuracy (13). Results of the present study demonstrated that the fern-like appearance was observed at a frequency of $90.0 \%$ in TSA, $36.4 \%$ in SSA and $8.3 \%$ in HP. In SSA, the serrated architecture is apparent in the basilar, but not the upper crypt regions $(2,16,17)$, rendering it difficult to obtain the characteristic pit pattern as observed in TSA. However, the pit pattern was only assessed in 33 lesions of our cohort, making the statistical analysis somewhat imprecise. Further studies in a larger number of serrated colorectal lesions are required.

This study is the first to examine the endoscopic features of SSA. Although not so accurate, we have found that the endoscopic discrimination of TSA was, to some extent, 
possible on the basis of conventional and magnified images, as shown in the literature (10-13). In contrast, discrimination of SSA from other serrated lesions may be difficult, although it is facilitated by its larger size and preferential location in the right colon. Recently developed imaging technology, such as narrow-band or autofluorescence imaging (21), may improve or mitigate this limitation and produce high diagnostic accuracy in differentiating serrated lesions.

Although our results were somewhat beneficial, a limitation of this study was that its design constituted of a retrospective single-center analysis comprised exclusively of Japanese patients. Therefore, our findings cannot be generalized to other institutes or other ethnic groups. Consequently, investigation into the context of multiple populations of patients and ethnic groups should be conducted, particularly in prospective studies.

In conclusion, endoscopic discrimination between SSA and other sessile serrated lesions based on morphological features was difficult. However, the size and location of the lesions facilitated diagnosis.

\section{Acknowledgements}

We thank Dr Yoichi Ajioka (Division of Molecular and Diagnostic Pathology, Niigata University) and Dr Jun Akiba (Department of Pathology, Kurume University) for their help during the study.

\section{References}

1. Longacre TA and Fenoglio-Preiser CM: Mixed hyperplastic adenomatous polyps/serrated adenomas. A distinct form of colorectal neoplasia. Am J Surg Pathol 14: 524-537, 1990.

2. Torlakovic E, Skovlund E, Snover DC, Torlakovic G and Nesland JM: Morphologic reappraisal of serrated colorectal polyps. Am J Surg Pathol 27: 65-81, 2003.

3. Snover DC, Jass JR, Fenoglio-Preiser C and Batts KP: Serrated polyps of the large intestine: a morphologic and molecular review of an evolving concept. Am J Clin Pathol 124: 380-391, 2005.

4. Jass JR: Hyperplastic polyps and colorectal cancer: is there a link? Clin Gastroenterol Hepatol 2: 1-8, 2004.

5. Farris AB, Misdraji J, Srivastava A, et al: Sessile serrated adenoma: challenging discrimination from other serrated colonic polyps. Am J Surg Pathol 32: 30-35, 2008
6. Iino H, Jass JR, Simms LA, et al: DNA microsatellite instability in hyperplastic polyps, serrated adenomas, and mixed polyps: a mild mutator pathway for colorectal cancer? J Clin Pathol 52: 5-9, 1999

7. O’Brien MJ, Yang S, Clebanoff JL, et al: Hyperplastic (serrated) polyps of the colorectum: relationship of $\mathrm{CpG}$ island methylator phenotype and K-ras mutation to location and histologic subtype. Am J Surg Pathol 28: 423-434, 2004.

8. O'Brien MJ, Yang S, Mack C, et al: Comparison of microsatellite instability, $\mathrm{CpG}$ island methylation phenotype, BRAF and KRAS status in serrated polyps and traditional adenomas indicates separate pathways to distinct colorectal carcinoma end points. Am J Surg Pathol 30: 1491-1501, 2006.

9. Spring KJ, Zhao ZZ, Karamatic R, et al: High prevalence of sessile serrated adenomas with BRAF mutations: a prospective study of patients undergoing colonoscopy. Gastroenterology 131: 1400-1407, 2006.

10. Matsumoto T, Mizuno M, Shimizu M, Manabe T, Iida M and Fujishima M: Serrated adenoma of the colorectum: colonoscopic and histologic features. Gastrointest Endosc 49: 736-742, 1999.

11. Jaramillo E, Watanabe M, Rubio C and Slezak P: Small colorectal serrated adenomas: endoscopic findings. Endoscopy 29: 1-3, 1997.

12. Oka S, Tanaka S, Hiyama T, et al: Clinicopathologic and endoscopic features of colorectal serrated adenoma: differences between polypoid and superficial types. Gastrointest Endosc 59: 213-219, 2004.

13. Sano Y, Saito Y, Fu K, et al: Efficacy of magnifying chromoendoscopy for the differential diagnosis of colorectal lesions. Dig Endosc 17: 105-116, 2005.

14. Kudo S, Hirota S, Nakajima T, et al: Colorectal tumours and pit pattern. J Clin Pathol 47: 880-885, 1994.

15. Tobaru T, Mitsuyama K, Tsuruta O, Kawano H and Sata M: Sub-classification of type VI pit patterns in colorectal tumors: Relation to the depth of tumor invasion. Int J Oncol 33: 503-508, 2008.

16. Torlakovic E and Snover DC: Serrated adenomatous polyposis in humans. Gastroenterology 110: 748-755, 1996.

17. Torlakovic EE, Gomez JD, Driman DK, et al: Sessile serrated adenoma (SSA) vs. traditional serrated adenoma (TSA). Am J Surg Pathol 32: 21-29, 2008.

18. Goldstein NS, Bhanot P, Odish E and Hunter S: Hyperplastic-like colon polyps that preceded microsatellite-unstable adenocarcinomas. Am J Clin Pathol 119: 778-796, 2003.

19. Lazarus R, Junttila OE, Karttunen TJ and Makinen MJ: The risk of metachronous neoplasia in patients with serrated adenoma. Am J Clin Pathol 123: 349-359, 2005.

20. Young J, Jenkins M, Parry S, et al: Serrated pathway colorectal cancer in the population: genetic consideration. Gut 56: 1453-1459, 2007.

21. Basu S, Torigian D and Alavi A: The role of modern molecular imaging techniques in gastroenterology. Gastroenterology 135: 1055-1061, 2008. 